\title{
SOME STATISTICAL PROBLEMS ENCOUNTERED IN A THEORY OF PINNING AND BREAK AWAY OF DISLOCATIONS*
}

\author{
BY \\ G. F. NEWELL \\ Brown University
}

1. Introduction. From a physical model proposed by Granato and Lücke [1] to describe the mechanical hysteresis in crystals caused by the motion of pinned dislocation lines, the following type of statistical problems arise. One distributes points (impurity atoms) at random along a line (edge dislocation) according to a Poisson distribution and then extracts from this line, segments of length $Z$, a random variable having a probability density $P(Z)$. We wish to calculate:

(1) the probability that in this segment, no pair of second nearest neighbor points are separated by a distance greater than some preassigned distance $D$; the ends of the segments are treated as additional points on the same basis as the randomly distributed ones.

(2) the probability distribution for the distance between consecutive points (again including the ends of the segment as additional points) for those configurations of points satisfying the condition given in (1).

For a detailed discussion of the origin and physical significance of this problem in relation to the theory of dislocation lines, the reader is referred to the work of Granato and Lücke which deals mainly with the physical aspects of hysteresis in solids. Although the connection between the above more abstract formulation of the problem and the physical model described by them will be outlined briefly, the main emphasis here will be on the mathematical solution. We shall obtain solutions in the form of single contour integrals involving the Laplace transform of $P(Z)$. In some cases, particularly if $P(Z)$ is exponential (Poisson distribution of $Z$ ) the solution is obtained exactly in closed form.

A dislocation line is an imperfection in the crystal. The physical details of its structure are not important to the present discussion; suffice it to say that it behaves in most respects like an elastic string under tension. Displacement of the dislocation line is inhibited by impurity atoms and by intersections with other dislocation lines so that under stress the dislocation line is pinned at various points along its length and is deformed much like an elastic string fastened at many points. A distinction is to be made between "weak pins" represented by the impurity atoms and "strong pins" represented by the crossing dislocation lines. The weak pins are capable of holding the dislocation line only if the force exerted on them is less than some maximum value. If the force exceeds this maximum, the string breaks away from the pin. The strong pins, on the other hand, fasten the dislocation line under arbitrary stress.

If a dislocation line is placed in a uniform stress field which exerts a certain lateral force per unit length of line, this force is transmitted to the pinning points. Each pin must support half of the force exerted on the two pieces of dislocation line on either

*Received July 30,1957 . The work described here was supported, in part, by the United States Army Signal Corps and by a grant from the Alfred P. Sloan Foundation. 
side of it. The force on the pin is therefore proportional to the distance between its two nearest neighbor pinning points, the total length of line that this pin must help support.

The dislocation line breaks away from the weak pin if this force exceeds a certain critical value or if the distance between its two neighbors on either side is greater than some critical distance $D$ which is inversely proportional to the force per unit length of dislocation line. If for any given stress a weak pin should break, its load must be supported by the adjacent pins. If the adjacent pins are also weak pins, they also break for they must now attempt to support a still longer length of line than the pin that broke. The result is that all weak pins on either side will break until the line is finally supported by the two nearest strong pins on either side. See Fig. 1 .

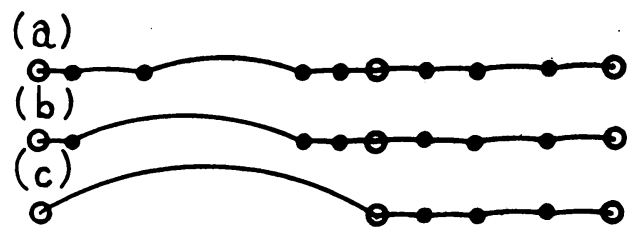

Fig. 1. (a) Shows a dislocation line under small stress with no break away. The circles denote strong pins, the dots weak pins and the line is supported at all pins.

(b) Shows an intermediate and unstable stage in the break away. The weak pin supporting the largest total length of line was broken.

(c) Shows the final stage of break away in which the line is supported only by the strong pins for that segment in which break away has occurred.

If, therefore, in a segment between two strong pins, there is any weak pin supporting pieces of the dislocation line having total length greater than $D$, all the weak pins in that segment break and the segment is entirely supported by the strong pins. Equivalently all weak pins between two strong pins break if any pair of second nearest neighbor pins are separated by a distance greater than $D$.

If weak pins and strong pins were spaced at regular intervals along the line, then for any given stress, we would find that either all weak pins in all segments broke or none broke. This would give rise to a sudden change in the physical behavior of the system at some critical stress, a situation which is not entirely satisfactory from the physical point of view. If, on the other hand, the pinning points were distributed according to some statistical law, there would be a certain non-zero probability that some of the weak pins would break for any value of the stress. This type of model leads to a hysteresis effect which, though strongly dependent upon the amplitude of the stress field, does not vary discontinuously.

Granato and Lücke suggest that a reasonable assumption would be that weak pins are distributed at random along the dislocation line, i.e. according to a Poisson distribution. Because of interactions between strong pins, however, they proposed to consider the distances between strong pins as independent random variables, not necessarily of Poisson type. They suggest, in fact, that it might be desirable to consider a network of strong pins spaced at equal intervals. We will consider an arbitrary probability density $P(Z)$ for the distance $Z$ between strong pins but will find that a Poisson distribution for $Z$ leads to the simplest results mathematically.

The questions that now arise are the following:

1. What is the probability that in any segment of line between two strong pins, no 
break away has occurred for some given stress or equivalently for some given value of the critical distance $D$ ?

2. What is the probability distribution for the distance between consecutive unbroken pins for any given value of $D$ ?

These two problems are the same problems as those described at the beginning of this section except they are stated in a different form. The second problem above can be decomposed into two parts; to find the distribution of distances if break away has not occurred and that if break away has occurred. The former is equivalent to the previous problem 2 whereas the answer to the latter follows directly from the fact that the distance in question is $Z$ having a given probability density $P(Z)$.

For the subsequent calculations, we introduce the following notation (see Fig. 2):

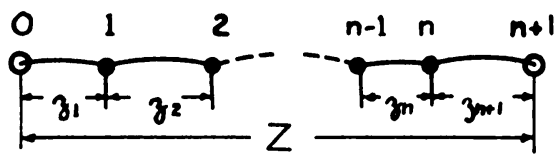

Frg. 2. In a segment containing $n$ weak pins, points are numbered consecutively from 0 to $n+1$ with the ends (strong pins) numbered 0 and $n+1$. Distances between points are denoted by $z_{1}, \ldots, z_{n+1}$ and the total length is given by $Z$.

$n$ is a random variable designating the number of points (weak pins) within some segment of length $Z$ (between strong pins);

$z_{j}$ denotes the distance between consecutive points with $z_{1}$ the distance from the end of the segment to the first point and $z_{n+1}$ the distance from the $n$th point to the other end of the segment;

$l$ is the average distance between randomly distributed points; $l^{-1}$ is the density of weak pins.

From the definition of $z_{i}$, it follows that

$$
Z=\sum_{i=1}^{n+1} z_{i}
$$

and from the assumption that the weak pins are distributed according to a Poisson distribution with density $l^{-1}$, it follows that $l^{-1} \exp \left(-z l^{-1}\right) d z$ is the a priori probability that the distance $z$ between any two consecutive weak pins lies in the interval between $z$ and $z+d z$. The probability, for fixed $Z$, that there are $n$ points in $Z$ and $z_{1}$ has a value between $z_{1}$ and $z_{1}+d z_{1}, z_{2}$ has a value between $z_{2}$ and $z_{2}+d z_{2}$ etc. and there are no points in the last interval of length $z_{n+1}=Z-\sum_{1}^{n} z_{i}$, is

$$
\begin{gathered}
l^{-n} \exp \left(-l^{-1} \sum_{i=1}^{n+1} z_{i}\right) d z_{1} \cdots d z_{n}=l^{-n} \exp \left(-l^{-1} Z\right) \prod_{i=1}^{n} d z_{i}, \quad \text { for } n>0 \\
\exp \left(-l^{-1} z_{1}\right)=\exp \left(-l^{-1} Z\right), \quad \text { for } n=0 .
\end{gathered}
$$

Since $P(Z)$ is the preassigned probability density of $Z=\sum_{i=1}^{n+1} z_{i}$, we obtain the following for the probability that the interval is of length $Z$ with $z_{1}$ between $z_{1}$ and $z_{1}+d z_{1}, \cdots, z_{n+1}$ between $z_{n+1}$ and $z_{n+1}+d z_{n+1}$ :

$$
l^{-n} P(Z) \exp \left(-Z l^{-1}\right) d z_{1} d z_{2} \cdots d z_{n+1}, \quad(n \geq 0) \text {. }
$$


The prescription for evaluating the average value $\langle f\rangle$ of any function $f\left(n, z_{1}, z_{2}\right.$, $\left.\cdots, z_{n+1}\right)$ of the distribution of pins is to compute

$$
\langle f\rangle=\sum_{n=0}^{\infty} \int_{0}^{\infty} d z_{1} \int_{0}^{\infty} d z_{2} \cdots \int_{0}^{\infty} d z_{n+1} P(Z) l^{-n} \exp \left(-Z l^{-1}\right) f\left(n, z_{1}, \cdots, z_{n+1}\right) .
$$

It will usually be impossible to evaluate multiple integrals of this type unless $f$ is of a special form. Fortunately, the quantities of interest here can be represented by products of relatively simple factors or sums of such products.

2. The probability of no break away. The first problem is to find the probability $p$ that no pair of second nearest neighbors in some segment are separated by a distance greater than $D$, i.e. the probability that $z_{j}+z_{i+1}<D, j=1,2, \cdots, n$. This is equivalent to finding the average of a function $f$ which has the value 1 if $z_{j}+z_{i+1}<D$ for all $j$ and 0 if $z_{i}+z_{i+1}>D$ for any $j$. Such a function can be written in the form

$$
g\left[\left(z_{1}+z_{2}\right) / D\right] g\left[\left(z_{2}+z_{3}\right) / D\right] \cdots g\left[\left(z_{n}+z_{n+1}\right) / D\right],
$$

in which the function $g$ is defined by

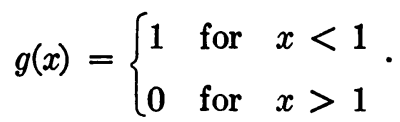

In view of the form of (3), it is convenient to measure $z$ in units of $D$ by writing

$$
x_{i}=z_{i} / D
$$

so that substitution of (3) into Eq. (2) for $f$ gives

$$
\begin{aligned}
& p=\sum_{n=0}^{\infty} D(D / l)^{n} \int_{0}^{\infty} d x_{1} \cdots \int_{0}^{\infty} d x_{n+1} P\left(D \sum_{i=1}^{n+1} x_{i}\right) \exp \left(-D l^{-1} \sum_{i=1}^{n+1} x_{i}\right) \\
& \times \prod_{i=1}^{n} g\left(x_{i}+x_{i+1}\right) .
\end{aligned}
$$

In these integrals, only the function $P$ fails to be in a form that can be factored into a product of functions of only one or two integration variables. We can obtain a factored form of the integrand at the expense of an extra integration by using the Laplace transform of $P$. If we define

$$
A(s) \equiv \int_{0}^{\infty} d Z e^{-\bullet z} P(Z)
$$

then

$$
P(Z)=\frac{1}{2 \pi i} \int_{\gamma-i \infty}^{\gamma+i \infty} d s e^{i z} A(s),
$$

in which $\gamma$ is some real number for which $A(\gamma)$ is defined by Eq. (6a). Equation (6b) is the well-known formula for inverting the Laplace transform.

If we substitute Eq. (6b) for $P(Z)$ in Eq. (5), let

$$
\alpha \equiv\left(s-l^{-1}\right) D,
$$

and interchange some integrations and summations, we obtain 


$$
\begin{aligned}
p & =\frac{1}{2 \pi i} \int_{\gamma-i \alpha}^{\gamma+i \alpha} d \alpha A\left(\alpha D^{-1}+l^{-1}\right) \sum_{n=0}^{\infty}(D / l)^{n} \int_{0}^{\infty} d x_{1} e^{\alpha x_{1}} \\
& \times \int_{0}^{\infty} d x_{2} e^{\alpha x_{2}} g\left(x_{1}+x_{2}\right) \cdots \int_{0}^{\infty} d x_{n} e^{\alpha x_{n}} g\left(x_{n-1}+x_{n}\right) \int_{0}^{\infty} d x_{n+1} e^{\alpha x_{n}+1} g\left(x_{n}+x_{n+1}\right),
\end{aligned}
$$

in which $\gamma$ must now be chosen so that $A\left(l^{-1}+\gamma D^{-1}\right)$ exists as defined by Eq. (6a).

The multiple integrals of Eq. (8) are in such a form that most of the integrals represent only a repetition of the single integral operation. Equation (8) can be simplified, formally at least, by introducing an integral operator $\mathbf{K}$ defined by the equation

$$
\mathbf{K} f(x) \equiv \int_{0}^{\infty} d x^{\prime} e^{\alpha x^{\prime}} g\left(x+x^{\prime}\right) f\left(x^{\prime}\right),
$$

for any function $f(x)$. In terms of this operator $\mathbf{K}$, Eq. (8) takes the form

$$
p=\frac{1}{2 \pi i} \int_{\gamma-i \infty}^{\gamma+i \infty} d \alpha A\left(\alpha D^{-1}+l^{-1}\right) \int_{0}^{\infty} d x e^{\alpha x} \sum_{n=0}^{\infty}(D / l)^{n} \mathbf{K}^{n} \mathbf{I} .
$$

$\mathbf{K}^{n} \mathbf{I}$ denotes $n$ repetitions of the operator $\mathbf{K}$ on the function $f(x)=1$.

The sum orer $n$ in Eq. (10) can be formally written as

$$
\sum_{n=0}^{\infty}(D / l)^{n} \mathbf{K}^{n}=(\mathbf{I}-\mathbf{K} D / l)^{-1} .
$$

Thus the problem of evaluating the sum of multiple integrals in Eq. (8), reduces to an investigation of the properties of the operator $(\mathrm{I}-\mathrm{K} D / l)^{-1}$.

The operator $(\mathbf{I}-\mathbf{K} D / l)$ is well defined by Eq. (9) and transforms any function $\psi(x)$ upon which it operates into a function $\varphi(x)$ according to the rule,

$$
\varphi(x)=(\mathbf{I}-\mathbf{K} D / l) \psi(x)=\psi(x)-(D / l) \int_{0}^{\infty} d x^{\prime} e^{\alpha x^{\prime}} g\left(x+x^{\prime}\right) \psi\left(x^{\prime}\right) .
$$

The operator $(\mathbf{I}-\mathbf{K} D / l)^{-1}$ must describe the inverse transformation

$$
\psi(x)=(\mathbf{I}-\mathbf{K} D / l)^{-1} \varphi(x) .
$$

In other words Eq. (11) applied to any function $\varphi(x)$ produces the solution $\psi(x)$ of the integral equation, Eq. (12). We see that for Eq. (10) we wish to know the function $\psi(x)$ resulting from $\varphi(x)=1$. For future reference, however, we shall solve Eq. (12) for arbitrary $\varphi(x)$.

Substitution of the explicit form for $g(x)$, Eq. (4), into Eq. (12) gives

$$
\begin{gathered}
\varphi(x)=\psi(x) \text { for } x>1 \\
\varphi(x)=\psi(x)-(D / l) \int_{0}^{1-x} d x^{\prime} e^{\alpha x^{\prime}} \psi\left(x^{\prime}\right) \text { for } x<1 .
\end{gathered}
$$

Equation (14a) needs no further study. Equation (14b) can be transformed into a familiar type of differential equation by first differentiating with respect to $x$ to obtain,

$$
\frac{d \varphi(x)}{d x}=\frac{d \psi(x)}{d x}+\frac{D}{l} e^{\alpha(1-x)} \psi(1-x) .
$$

Replacement of $x$ by $1-x$ gives

$$
-\frac{d \varphi(1-x)}{d x}=-\frac{d \psi(1-x)}{d x}+\frac{D}{l} e^{\alpha x} \psi(x) .
$$


By solving Eq. (15a) for $\psi(1-x)$ and eliminating it from Eq. (15b), one obtains the equation

$$
\frac{d^{2} \psi(x)}{d x^{2}}+\alpha \frac{d \psi(x)}{d x}+\left(\frac{D}{l}\right)^{2} e^{\alpha} \psi(x)=\frac{d^{2} \varphi(x)}{d x^{2}}+\alpha \frac{d \varphi(x)}{d x}-\frac{D}{l} e^{\alpha(1-x)} \frac{d \varphi(1-x)}{d x} .
$$

This equation to find $\psi$ in terms of $\varphi$ is the famous linear inhomogeneous differential equation with constant coefficients, the formal solution of which is elementary. For the boundary conditions, we observe from Eqs. (14b) and (15a) that

$$
\begin{aligned}
\psi(1) & =\varphi(1) \\
\left.\frac{d \psi(x)}{d x}\right|_{x=0} & =-\frac{D}{l} e^{\alpha} \varphi(1)+\left.\frac{d \varphi(x)}{d x}\right|_{x=0} .
\end{aligned}
$$

Actually it is not necessary to find the complete solution of these equations. All we need in Eq. (10) is the value of the integral

$$
\begin{aligned}
\int_{0}^{\infty} d x e^{\alpha x} \psi(x) & =\int_{1}^{\infty} d x e^{\alpha x} \psi(x)+\int_{0}^{1} d x e^{\alpha x} \psi(x) \\
& =\int_{1}^{\infty} d x e^{\alpha x} \varphi(x)+[\psi(0)-\varphi(0)](l / D)
\end{aligned}
$$

by virtue of Eq. (14a) and (14b). Thus we need find only $\psi(0)$ evaluated for $\varphi(x)=1$.

The explicit evaluation of the indicated steps is straightforward and leads to the result

$$
p=\frac{1}{2 \pi i} \int_{\gamma-i \infty}^{\gamma+i \infty} d \alpha A\left(\alpha D^{-1}+l^{-1}\right)\left\{\frac{e^{-a}-e^{-b}+(b-a)(l / D)}{b e^{a}-a e^{b}}-\frac{l}{D}-\frac{e^{\alpha}}{\alpha}\right\},
$$

where $a$ and $b$ are the two roots of the equation

$$
\left.\begin{array}{l}
a \\
b
\end{array}\right\}=-\left(\frac{\alpha}{2}\right) \pm\left[\left(\frac{\alpha}{2}\right)^{2}-\left(\frac{D}{l}\right)^{2} e^{\alpha}\right]^{1 / 2} \text {. }
$$

We previously imposed a restriction on $\gamma$ which is equivalent to requiring that the path of integration from $\gamma-i \infty$ to $\gamma+i \infty$ in Eq. (18) lies to the right of all singularities of $A\left(\alpha D^{-1}+l^{-1}\right)$. The expression in the bracket of Eq. (18) also has singularities, infinitely many of them, and they arise originally from the fact that the sum over $n$ in Eq. (8) will not necessarily converge if the real part of $\alpha$ is too large. To justify the various formal steps leading to Eq. (18) we must be sure that the series converges for all $\alpha$ along the path of integration. This is achieved by picking the path to the left of all singularities of the expression in the bracket. It is always possible to pick such a path. One can show for example that $\gamma=-D / l$ is always satisfactory.

At this point, we could express $p$ directly in terms of the given probability distribution $P(Z)$ by using the definition of $A$, Eq. (6a), to eliminate $A(s)$ from Eq. (18) in favor of $P(Z)$. This would give a double integral over both $\alpha$ and $Z$ which would be very difficult to reduce to simpler form. The integral over $Z$ cannot be done unless $P(Z)$ is explicitly specified and the integral over $\alpha$ is too complicated to perform first. We cannot conveniently proceed further unless we specify the form of $P(Z)$ or equivalently its transform $A(s)$.

Despite the complexity of Eq. (18), there is a sizeable class of functions $P(Z)$ or 
transforms $A(s)$ for which this final integration can be performed explicitly. The trick is to consider functions $A(s)$ having only a finite number of poles, to close the contour in Eq. (18) with a semi-circle in the left half plane and use residue theory. One then avoids the complicated singularities of the integrand in the right half plane.

The class of functions $P(Z)$ which can be treated in this way include exponentials, polynomials in $Z$ times exponentials or any linear combination of such. One could at least approximate any $P(Z)$ with functions in this class but the analysis would be extremely tedious for any but the simplest examples.

If we have a Poisson distribution of strong pins, i.e.

$$
P(Z)=L^{-1} e^{-Z / L}
$$

with $L$ the average distance between strong pins; $L^{-1}$ the density, then

$$
A\left(\alpha D^{-1}+l^{-1}\right)=D L^{-1}\left[\alpha+D l^{-1}+D L^{-1}\right]^{-1} .
$$

In Eq. (18) there is only one singularity to the left of the contour, namely the simple pole of $A$ at $\alpha=-D l^{-1}-D L^{-1}$. The value of $p$ is found explicitly as

$$
p=\frac{D}{L}\left\{\frac{e^{-a}-e^{-b}+(b-a)(l / D)}{b e^{a}-a e^{b}}-\frac{l}{D}-\frac{e^{\alpha}}{\alpha}\right\}
$$

with

$$
\left.\begin{array}{l}
a \\
b
\end{array}\right\}=-\left(\frac{\alpha}{2}\right) \pm\left[\left(\frac{\alpha}{2}\right)^{2}-\left(\frac{D}{l}\right)^{2} e^{\alpha}\right]^{1 / 2}
$$

and

$$
\alpha=-D\left(l^{-1}+L^{-1}\right) .
$$

The probability $p$ is a function of only two independent variables $D / l$, the ratio of the break away length to the average distance between weak pins and $D / L$, the ratio of the break away length to the average distance between strong pins. The values of these two ratios determine $\alpha, a$, and $b$ as given by Eqs. (21b) and (21c) from which $p$ is evaluated.

A discussion of these results and some approximations derived from them will be described in Sec. 4.

3. Average of additive functions. Most practical problems are concerned with finding the average value of some function which is the sum of contributions from each of the intervals of dislocation line between consecutive pins. We therefore desire to compute the average $\langle G\rangle$ of a function of the form

$$
G\left(n, z_{1}, z_{2}, \cdots, z_{n}\right)=\left\{\begin{aligned}
& G\left(z_{1}\right)+G\left(z_{2}\right)+\cdots+G\left(z_{n+1}\right) \\
& \text { if } z_{j}+z_{i+1}<D \text { for all } j \\
& G(Z) \text { if } z_{j}+z_{i+1}>D \text { for any } j .
\end{aligned}\right.
$$

This class of functions includes as special cases the following:

1. If $G(z)=z$, then both Eq. (22a) and Eq. (22b) have the value $Z$ and $\langle G\rangle=\langle Z\rangle \equiv L$ independent of $D$.

2. If $G(z)=1$, Eqs. (22a) and (22b) give the values $n+1$ and 1 respectively. The value of $\langle G\rangle-1$ is therefore the average number of unbroken weak pins per segment. 
3. If $G(z)=\delta\left(z-z_{0}\right)$ then $\langle G\rangle / \int_{0}^{\infty}\langle G\rangle d z_{0}$ is the probability density for the distance between consecutive unbroken pins. The evaluation of this was listed in Sec. 1 as one of the problems of particular concern, Knowing this for arbitrary $z_{0}$ we could in fact go back and evaluate $\langle G\rangle$ for arbitrary $G$ by superposition. We shall treat the problem of finding $\langle G\rangle$ for arbitrary functions directly, however, since the determination of the probability density for the distance between unbroken pins is usually of no direct physical importance itself but only a means of finding the average of certain additive functions.

Of particular interest to the theory of mechanical hysteresis is the case $G(z)=z^{3}$. The displacements of dislocation lines contribute to the strain and consequently also to the elastic coefficients. For the loading portion of the stress-strain curve, this contribution to the elastic coefficient is proportional to the sum of cubes of the interval lengths, i.e. to $\langle G\rangle$ with $G(z)=z^{8}$. The stress-strain curve for loading is non-linear because $\langle G\rangle$ depends upon the break away distance $D$ which in turn is inversely proportional to the stress. As more pins break with increasing strain, the ratio of strain to stress increases. The unloading portion of the stress-strain curve is linear, however, because those pins that are broken on the loading portion of the curve remain broken during the unloading.

With the aid of Eq. (4) we can write Eq. (22) in the form

$$
G\left(n, z_{1}, \cdots, z_{n+1}\right)=G(Z)-\left[G(Z)-\sum_{i=1}^{n+1} G\left(z_{j}\right)\right] \prod_{k=1}^{n} g\left[\left(z_{k}+z_{k+1}\right) / D\right] .
$$

$\langle G\rangle$ can then be split into three parts by writing

$$
\langle G\rangle=\left\langle G_{1}\right\rangle+\left\langle G_{2}\right\rangle-\left\langle G_{3}\right\rangle
$$

with

$$
\begin{aligned}
&\left\langle G_{1}\right\rangle=\sum_{n=0}^{\infty} \int_{0}^{\infty} d z_{1} \cdots \int_{0}^{\infty} d z_{n+1} P(Z) l^{-n} \exp (-Z / l) \times\left[\sum_{i=1}^{n+1} G\left(z_{j}\right)\right] \prod_{k=1}^{n} g\left[\left(z_{k}+z_{k+1}\right) / D\right] \\
&\left\langle G_{2}\right\rangle=\int_{0}^{\infty} d Z P(Z) G(Z) \\
&\left\langle G_{3}\right\rangle=\sum_{n=0}^{\infty} \int_{0}^{\infty} d z_{1} \cdots \int_{0}^{\infty} d z_{n+1} P(Z) l^{-n} \exp (-Z / l) G(Z) \prod_{k=1}^{n} g\left[\left(z_{k}+z_{k+1}\right) / D\right] .
\end{aligned}
$$

$\left\langle G_{1}\right\rangle$ and $\left\langle G_{2}\right\rangle-\left\langle G_{3}\right\rangle$ give respectively the contributions from pins that do not break and those which do break.

In view of the fact that neither $P(Z)$ or $G(Z)$ is explicitly specified, Eq. (23b) cannot be simplified any further. $\left\langle G_{3}\right\rangle$ is easily handled because Eq. (23c) and Eq. (5) differ only in that Eq. (23c) has $P(Z) G(Z)$ where Eq. (5) has $P(Z)$. Since $P(Z)$ is arbitrary, we can evaluate $\left\langle G_{3}\right\rangle$ simply by replacing $P(Z)$ in the formula for $p$ by $P(Z) G(Z)$ or equivalently by replacing the transform $A(s)$, Eq. (6), by

$$
B(s)=\int_{0}^{\infty} d Z e^{-s z} P(Z) G(Z) .
$$

We can also write $\left\langle G_{\vartheta}\right\rangle=B(0)$. 
Only $\left\langle G_{1}\right\rangle$ remains to be evaluated and this we do by a method which parallels that of the last section. In Eq. (23a), the summation over $j$ is taken outside the integrals; $z_{i}$ is replaced by $D x_{i} ; P(Z)$ is expressed in terms of $A(s)$; and the integrals are written in terms of the operator $\mathbf{K}$ noting that for any $j, G\left(z_{j}\right)$ appears only in the integral over $z_{i}$, all other integrals being of the same type as in the last section. This leads to the form

$$
\begin{aligned}
\left\langle G_{2}\right\rangle=\frac{1}{2 \pi i} \int_{\gamma-i \infty}^{\gamma+i \infty} d \alpha A\left(\alpha D^{-1}\right. & \left.+l^{-1}\right) \\
& \times \int_{0}^{\infty} d x_{1} e^{\alpha x_{2}}\left\{\sum_{n=0}^{\infty} \sum_{i=1}^{n+1}(D / l)^{n} \mathbf{K}^{i-1} G(D x) \mathbf{K}^{n-i+1} \mathbf{I}\right\} .
\end{aligned}
$$

The expression $\mathbf{K}^{i-1} G(D x) \mathbf{K}^{n-i+1} \mathbf{I}$ represents transformation of the function I with $\mathbf{K}, n-j+1$ times, multiplication of this function by $G(D x)$ and the transformation of the product with $\mathbf{K}, j-1$ times.

If we rearrange the order of summations by letting $k=j-1$ and $m=n-j+1$, the expression in the bracket can be written as

$$
\sum_{k=0}^{\infty} \sum_{m=0}^{\infty}(D / l)^{k} \mathbf{K}^{k} G(D x)(D / l)^{m} \mathbf{K}^{m} \mathbf{I}=(\mathbf{I}-\mathbf{K} D / l)^{-1} G(D x)(\mathbf{I}-\mathbf{K} D / l)^{-1} \mathbf{I} .
$$

The function $\psi(x)=(\mathbf{I}-\mathbf{K} D / l)^{-1} \mathbf{I}$ was encountered in the last section. It was also shown there how $(\mathbf{I}-\mathbf{K} D / l)^{-1} \varphi(x)$ could be found for any function $\varphi(x)$. This must be done for $\varphi(x)=G(D x) \psi(x)$ as required above and the integral over $x_{1}$ in Eq. (25) evaluated. This procedure leads to the final result that

$$
\begin{aligned}
\langle G\rangle= & B(0)-\frac{1}{2 \pi i} \int_{\gamma-i \infty}^{\gamma+i \infty} d \alpha B\left(\alpha D^{-1}+l^{-1}\right)\left\{\frac{e^{-a}-e^{-b}+(b-a) l / D}{\left(b e^{a}-a e^{b}\right)}-\frac{l}{D}\right\} \\
& +\frac{1}{2 \pi i} \int_{\gamma-i \infty}^{\gamma+i \infty} d \alpha A\left(\alpha D^{-1}+l^{-1}\right)\left(b e^{a}-a e^{b}\right)^{-2} \int_{0}^{1} d x G(D x) \\
& \times\left\{\left(b+e^{-a} D / l\right) \exp \left[\frac{1}{2}(a-b) x\right]-\left(a+e^{-b} D / l\right) \exp \left[-\frac{1}{2}(a-b) x\right]\right\}^{2} .
\end{aligned}
$$

The remaining integrations cannot be done explicitly unless the functions $P(Z)$ and $G(z)$ or $A(s)$ and $B(s)$ are specified. There is again an important class of functions $P(Z)$ and $G(Z)$ for which these last integrations can be performed analytically. They can be done if $P(Z)$ and $G(Z)$ are linear combinations of polynomials times exponentials (the same class of functions for which $p$ could be evaluated) and, in particular, if $G(z)$ is proportional to $z^{k}, k$ integer and $P(Z)$ a Poisson distribution. The trick is to close the contour of the $\alpha$ integral in the left half plane and use residue theory as done previously to obtain Eqs. (21) for $p$.

Although the evaluation of $\langle G\rangle$ in this latter case is straightforward, the residue of the first integral in Eq. (26) requires $k$ differentiations of the quantity in the bracket and the evaluation of the integral over $z$ in the last term of $\langle G\rangle$ involves integration of $z^{k}$ times exponentials, all of which are elementary operations but unfortunately ones which lead to very awkward formulas. We shall not give these formulas here.

4. Limiting cases. Although we have seen that it is possible to obtain results in closed form for many physically interesting properties of this model, the formulas are awkward to evaluate and it is highly desirable to obtain simple approximations capable of giving at least qualitatively correct results with a minimum of effort. 
For any particular material, the values of $l^{-1}$ and $L^{-1}$, the densities of weak and strong pins respectively, would be fixed but $D^{-1}$ which is proportional to the stress would be variable. The critical range of $D$ in which break away is most likely to occur will however depend upon $l^{-1}$ and $L^{-1}$ which themselves vary widely depending upon the material. For a very pure material we may find $l \gg L$, whereas for a less pure material we might have $L \gg l$. In either of these limiting cases, one can obtain simple approximations.

I. Pure materials. If $l \gg L$, there is a high probability that there will be no weak pins between two consecutive strong pins and therefore nothing to break. There is a small probability that there is one weak pin in the segment between strong pins and this pin would break or not break accordingly as $z_{1}+z_{2}=Z$ is greater or less than $D$. We could find $p$ directly from Eq. (5) by evaluating individual terms for $n=0,1, \ldots$. Since there is a very small probability for having more than one weak pin in a segment $(n>1)$, the first two terms of Eq. (5) would give a fairly good approximation.

A slightly better and in most cases simpler approximation results if we note that for $Z<D$, no break occurs for any value of $n$ nor can it occur for any $Z$ if $n=0$. We conclude that

$$
p>\int_{0}^{D} P(Z) d Z+\int_{D}^{\infty} P(Z) \exp (-Z / l) d Z,
$$

in which the first term represents the probability that $Z<D$ and the second term the probability that if $Z>D$ then $n=0$. We also can obtain a close upper bound by noting that the probability of break away is at least as great as the probability of break away for $n=1$, i.e.

$$
p<1-\int_{D}^{\infty} P(Z) \exp (-Z / l)(Z / l) d Z .
$$

These integrals can be evaluated for a wide variety of functions $P(Z)$. For example, if $P(Z)$ is Poisson then

$$
p\left\{\begin{array}{l}
>1-\exp (-D / L)+(1+L / l)^{-1} \exp [-(D / L)(1+L / l)] \\
<1-(L / l)(1+L / l)^{-2}[1+(D / L)(1+L / l)] \exp [-(D / L)(1+L / l)] .
\end{array}\right.
$$

If $P(Z)=\delta(Z-L)$, equal spacing of strong pins, then

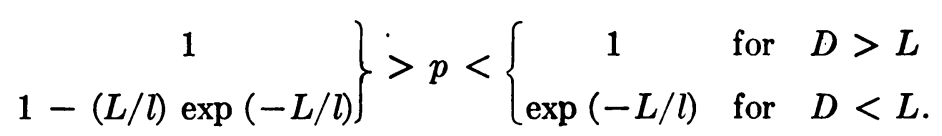

These bounds are valid for arbitrary values of $L / l$ but are close bounds (error of order $L^{2} / l^{2}$ ) only for $L / l \ll 1$. For large stress, $D \rightarrow 0$, and $L / l \ll 1$, Eqs. (27) and (28) show that $p-1 \sim L / l$ for any $P(Z)$. The probability of break away, $1-p$, is therefore small, of order $L / l$, because of the shortage of weak pins, but most of the break away that does occur takes place for $D$ of order $L$. The amount of break away is sensitive to $l / L$ but the stress at which it does occur is nearly independent of $l$ for $L / l \ll 1$.

For $L / l=1$, Fig. 3 shows the upper and lower bounds of $(1-p)$ given by Eqs. $(27 \mathrm{a}, \mathrm{b})$ and Eqs. $(28 \mathrm{a}, \mathrm{b})$, also the exact value of $1-p$ for the Poisson distribution of $Z$ given by Eq. (21). Even for $L / l=1$, Eq. (27a), the upper bound on $1-p$, is a reasonable approximation. The accuracy of Eq. (27a) is, of course, better for smaller values 


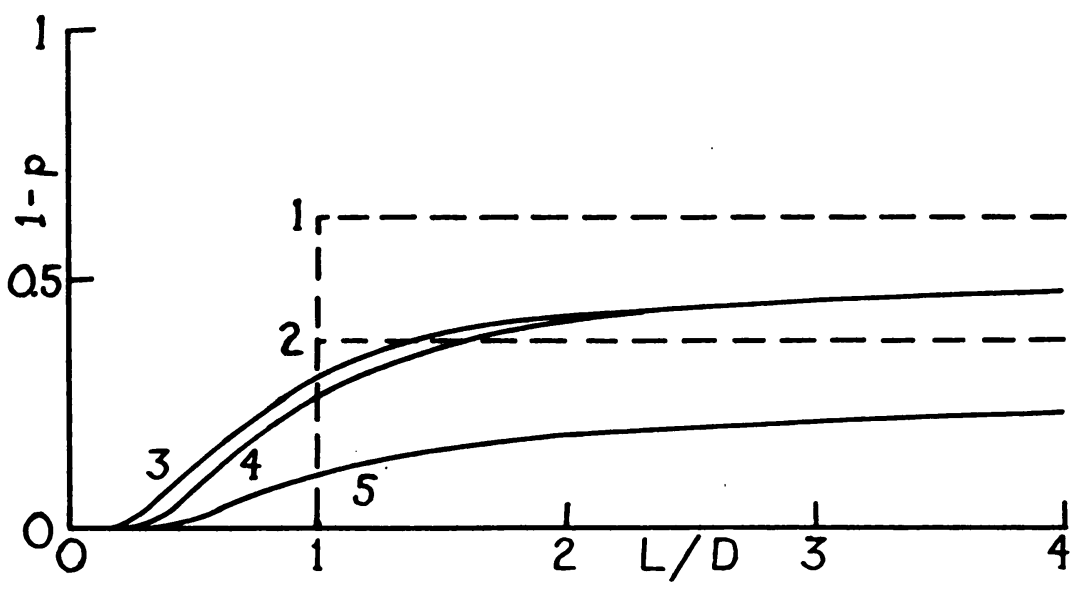

Frg. 3. The probability of break away $1-p$ is plotted as a function of $L / D$ (the stress) for $L / l=1$. Broken lines 1 and 2 are the upper and lower bounds on $1-p$ for equal spacing of strong pins, Eqs. (27b) and (28b). Curves 3, 5 and 4 are upper bounds, lower bounds and the exact solution respectively for $1-p$ with an exponential distribution of strong pins Eqs. (27a), (28a) and (21).

of $L / l$ but even for large values of $L / l$, Eq. (27a) is accurate in the limit of small $D$ (large $L / D$ ).

The value of $\langle G\rangle$ can be approximated using similar arguments. It is clear, first of all, that if there are very few weak pins, $L / l \rightarrow 0$, or if all the weak pins have broken, $D \rightarrow 0$, then the distance between unbroken pins is always $Z$ and

$$
\langle G\rangle \rightarrow \int_{0}^{\infty} d Z P(Z) G(Z) \text { for } L / l \rightarrow 0 \text { or } D \rightarrow 0 .
$$

If on the other hand, $D \rightarrow \infty$ (zero stress), nothing breaks and one can find $\langle G\rangle$ as a limiting case of Eq. (23) or Eq. (26). The result is

$$
\begin{array}{r}
\langle G\rangle \rightarrow \int_{0}^{\infty} d Z P(Z)\left\{G(Z) e^{-z / l}+l^{-2} \int_{0}^{Z} d z G(z)(Z-z+2 l) e^{-z / l}\right\} \\
\text { for } D \rightarrow \infty .
\end{array}
$$

If, in particular, we let $P(Z)=L^{-1} \exp (-Z / L)$, Eq. (29) gives directly

$$
\langle G\rangle \rightarrow L^{-1} \int_{0}^{\infty} d Z G(Z) e^{-Z / L}, \quad L / l \rightarrow 0 \text { or } D \rightarrow 0
$$

whereas Eq. (30) reduces to

$$
\langle G\rangle \rightarrow L^{-1}(1+L / l)^{2} \int_{0}^{\infty} d Z G(Z) \exp [-(Z / L)(1+L / l)] \text { for } D \rightarrow \infty .
$$

The similarity of Eqs. (29a) and (30a) results from the fact that if weak pins and strong pins separately have Poisson distributions, they combine to give a Poisson distribution of all pins with average density $L^{-1}(1+l / L)$.

As a further specialization we find from Eqs. (29a), (30a) that for $G(Z)=Z^{k}, k$ integer,

$$
\langle G\rangle \rightarrow k ! L^{k} \text { for } L / l \rightarrow 0 \text { or } D \rightarrow 0,
$$




$$
\langle G\rangle \rightarrow k ! L^{k}(1+L / l)^{1-k} \text { for } \quad D \rightarrow \infty .
$$

In each case, Eqs. (30), (30a), (30b) reduce to Eqs. (29), (29a), (29b) respectively if $L / l \rightarrow 0$.

To determine how $\langle G\rangle$ depends upon $D$ for $L / l \ll 1$ we may start from Eq. (29) and make a correction for the presence of a small number of unbroken weak pins or we can start from Eq. (30) and make a correction for a small number of broken pins. In either case we shall approximate the effects of unbroken or broken pins respectively by considering only the contributions for $n=1$, one weak pin between strong pins and neglect effects of $n>1$. This leads to the relations

$$
\begin{aligned}
& \langle G\rangle \sim\langle G\rangle_{D=0}+\int_{0}^{D} d Z P(Z) e^{-Z / l} l^{-1} \int_{0}^{Z} d x[2 G(x)-G(Z)] \\
& \langle G\rangle \sim\langle G\rangle_{D \rightarrow \infty}-\int_{D}^{\infty} d Z P(Z) e^{-Z / l} l^{-1} \int_{0}^{Z} d x[2 G(x)-G(Z)] .
\end{aligned}
$$

Both of these formulae are correct to order $L / l$ for all $D$ but Eq. (31) approaches the exact limit given by Eq. (29), for $D \rightarrow 0$ whereas Eq. (32) approaches the exact limit given by Eq. (30) for $D \rightarrow \infty$. If $P(Z)=\delta(Z-L),\langle G\rangle$ has a discontinuity at $D=L$ as did $p$. For the Poisson distribution, there is a more gradual change in the range $D$ of order $L$.

As a special case, we obtain

$$
\begin{gathered}
\langle G\rangle \sim k ! L^{k}\left\{1+(1-k)(L / l)(1+L / l)^{-(k+2)}[(k+1) !]^{-1} \int_{0}^{D(l-1+L-1)} d x e^{-x} x^{k+1}\right\} \\
\langle G\rangle \sim k ! L^{k}(1+L / l)^{1-k}\left\{1-(1-k)(L / l)(1+L / l)^{-3}[(k+1) !]^{-1}\right. \\
\left.\cdot \int_{D\left(l^{-1}+L^{-1}\right)}^{\infty} d x e^{-x} x^{k+1}\right\}
\end{gathered}
$$

if $P(Z)$ is Poisson and $G(Z)=Z^{k}$. The integrals above can either be evaluated directly or from tables of incomplete $\Gamma$-functions.

II. Impure materials. For $l \ll L$, it is clear that if $D$ is of order $L$, break away is nearly impossible because a negligible number of intervals between weak pins of average length $l$ will be of length comparable with $D$ as required for a break. On the other hand, if $D$ is of order $l$, break away is almost certain because there is a high probability that at least one of the many second neighbor distances will be greater than $D$. We conclude that the range of $D$ where break away is most likely to occur satisfies the condition

$$
l \ll D \ll L .
$$

By neglecting terms of order exp $(-D / l)$ compared with 1 in the equations of Sec. 2 , one finds that for an exponential distribution of $Z, E q$. (21) reduces to

$$
p \sim\left[1+\frac{L}{l}\left(\frac{D}{l}-1\right) e^{-D / l}\right]^{-1}
$$

and for most distributions $P\left(Z^{\prime}\right)$ one can use an approximation

$$
p \sim \int_{0}^{\infty} d Z P(Z) \exp \left[-\frac{Z}{l}\left(\frac{D}{l}-1\right) e^{-D / l}\right] .
$$


If all segments are of length $Z=L$, then

$$
p \sim \exp \left[-\frac{L}{l}\left(\frac{D}{l}-1\right) e^{-D / l}\right]
$$

which means that for $L / l \gg 1, p$ changes rapidly from 0 to 1 when $D / l$ is approximately of order $\log (L / l)$.

One can show from the equations of Sec. 2 or from physical arguments that for $L / l \gg 1$, break away does not appreciably distort the shape of the distribution of lengths between unbroken pins. The reason is that break away results mainly from some exceptionally long interval between a pair of weak pins. The existence of one such long interval does not significantly distort the distribution of lengths of the remaining intervals in $Z$. Yet when one pin breaks, they all break. The distribution of lengths between pins that break is therefore nearly the same as the distribution of lengths between pins that do not break.

To find $\langle G\rangle$ approximately we simply take $\langle G\rangle$ for the interval between two unbroken pins and multiply it by the average number of such intervals between strong pins if there is no break. We add to this $\langle G\rangle$ for the broken intervals to obtain

$$
\begin{aligned}
\langle G\rangle \sim\left\{\int_{0}^{\infty} d z G(z) l^{-1} e^{-s / l}\right. & \left\{\int_{0}^{\infty} d Z(1+Z / l) P(Z) \exp \left[-\frac{Z}{l}\left(\frac{D}{l}-1\right) e^{-D / l}\right]\right\} \\
& +\int_{0}^{\infty} d Z P(Z) G(Z)\left\{1-\exp \left[-\frac{Z}{l}\left(\frac{D}{l}-1\right) e^{-D / l}\right]\right\} .
\end{aligned}
$$

These integrals are easily evaluated for a wide variety of possible $G(z)$ and $P(z)$.

To illustrate the approximations of this section, we have plotted in Fig. $4,1-p$ vs. $L / D$ (break away vs. stress) for $P(Z)$ an exponential distribution with $L / l=100$.

5. Conclusions. We have found in Secs. 2 and 3 the exact solution of the problem described in Sec. 1 and obtained several limiting approximations. This problem is of

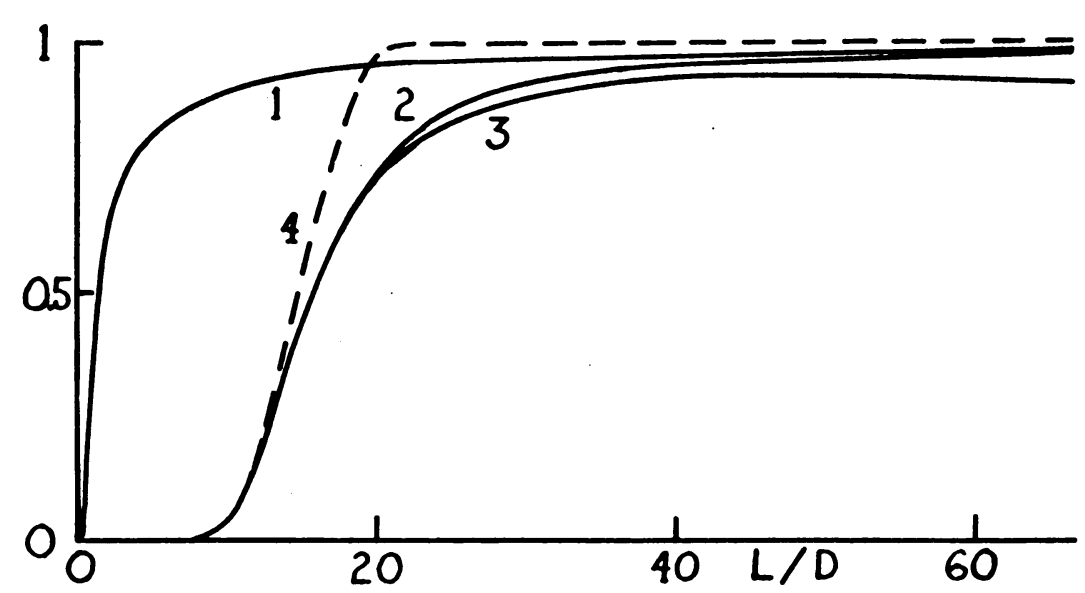

Fra. 4. The probability of break away, $1-p$, is plotted as a function of $L / D$ (the stress) for $L / l=$ 100 and an exponential distribution of strong pins. Curve 1 is the upper bound, Eq. (27a) [the corresponding lower bound Eq. (28a) is too small to draw on this scale]. Curve 2 is the exact solution, Eq. (21), and curve 3 is the approximation for $L / l \gg 1$, Eq. (34). Curve 4 (broken line) is the corresponding approximation for equal spacing of strong pins, Eq. (36). 
some practical importance to a field of physics that is still in a rather infant and uncertain stage of development but it also suggests a number of interesting mathematical problems that are somewhat different from the classic problems of probability theory.

None of the individual mathematical steps in the above analysis is new although they are borrowed from rather diverse origins. The analysis is formally quite similar to classic methods for dealing with Markov chains. If $P(Z)$ is exponential, the distribution of distances between second neighbor points can, in fact, be described in the form of a Markov chain. It would have been possible also to carry through most of the formal steps of Secs. 2 and 3 even if the $z_{j}$ were distributed according to a Markov chain rather than a Poisson distribution. One could, however, not expect to obtain explicit solutions in such cases.

Acknowledgments. The author is indebted to Professor Kurt Lücke and Dr. Andrew Granato for bringing this problem to his attention and for numerous very enlightening discussions particularly regarding the physical basis of various theories of dislocation line behavior and related topics.

\section{BibLIOGRAPHY}

1. A. Granato and K. Lücke, Theory of mechanical damping due to dislocations, J. Appl. Phys. 27, 58393 (1956) 